\title{
Intensive glycaemic control for patients with type 2 diabetes: systematic review with meta-analysis and trial sequential analysis of randomised clinical trials
}

In the final stages of production, the authors of this paper, Bianca Hemmingsen and colleagues, made some late changes to data in the 14 figures, resulting in some errors in the published article (BMJ 2011;343:d6898, doi:10.1136/bmj.d6898). Additionally, after publication they also noticed some further errors. In the abstract and results section, the $\mathrm{P}$ value for retinopathy should be 0.008 [rather than 0.009 ] and the number of participants should be 10070 [not 10 793]. The trial sequential analysis adjusted $95 \%$ confidence interval for retinopathy should be 0.55 to 1.15 [not 0.54 to 1.17 ]. Figure $10 \Downarrow$ and figure $11 \Downarrow$ have been corrected; and in the legend to fig 11 , the heterogeneity adjusted required information size is 40021 [not 43 960] participants calculated on the basis of proportion of retinopathy of $15.5 \%$ [not $14.3 \%$ ] in the conventional glucose control group, and the number of participants is as above. In table 2, the conventional glycaemic control column for Jaber et al 1996 and REMBO 2008 should read "Standard treatment" [rather than "Not specified"]. Table $4 \Downarrow$ contained incorrect information for selective outcome reporting bias and had been corrected. Finally, in appendix 3 of the webextra (original re-posted here), the retinopathy outcome for UGDP 1975 should be "Fundus abnormalities excluding exudates."

Cite this as: BMJ 2012;344:d8277

๑ BMJ Publishing Group Ltd 2012 


\section{Table}

\section{Table 4 (Corrected)| Risk of bias assessments of included trials}

\begin{tabular}{|c|c|c|c|c|c|c|}
\hline Trial & $\begin{array}{l}\text { Sequence } \\
\text { generation }\end{array}$ & $\begin{array}{c}\text { Allocation } \\
\text { concealment }\end{array}$ & Blinding & $\begin{array}{l}\text { Incomplete outcome } \\
\text { data }\end{array}$ & $\begin{array}{l}\text { Selective outcome } \\
\text { reporting }\end{array}$ & Free from other bias \\
\hline ACCORD $2008^{467-74}$ & Adequate & Adequate & Adequate & Unclear & Adequate & Inadequate \\
\hline ADVANCE $2008^{75-78}$ & Adequate & Adequate & Adequate & Adequate & Adequate & Inadequate \\
\hline Bagg et al $2001^{79-82}$ & Unclear & Unclear & Adequate & Adequate & Adequate & Adequate \\
\hline Becker et al $2003^{8384}$ & Unclear & Unclear & Unclear & Unclear & Unclear & Adequate \\
\hline IDA $2009^{9293}$ & Adequate & Adequate & Adequate & Adequate & Unclear & Inadequate \\
\hline Jaber et al $1996^{94}$ & Unclear & Unclear & Unclear & Adequate & Unclear & Inadequate \\
\hline Kumamoto $2000^{79596}$ & Unclear & Unclear & Unclear & Adequate & Unclear & Inadequate \\
\hline Lu et al $2010^{86}$ & Unclear & Unclear & Unclear & Unclear & Unclear & Adequate \\
\hline REMBO $2008^{85}$ & Unclear & Unclear & Unclear & Adequate & Unclear & Unclear \\
\hline Service et al $1983^{87}$ & Adequate & Unclear & Adequate & Adequate & Unclear & Adequate \\
\hline UGDP $1978^{88-91}$ & Adequate & Adequate & Adequate & Adequate & Adequate & Adequate \\
\hline UKPDS $1998^{1897-102}$ & Adequate & Adequate & Adequate & Adequate & Adequate & Inadequate \\
\hline VA CSDM $1995^{103-109}$ & Unclear & Unclear & Adequate & Adequate & Adequate & Inadequate \\
\hline VADT $2009^{6110111}$ & Adequate & Adequate & Adequate & Adequate & Adequate & Inadequate \\
\hline
\end{tabular}

ACCORD=Action to Control Cardiovascular Risk in Diabetes Study; ADVANCE=Action in Diabetes and Vascular disease-PreterAx and DiamicroN MR Controlled Evaluation; IDA=Insulin Diabetes Angioplasty; REMBO=Rational Effective Multicomponent Therapy in the Struggle Against DiaBetes Mellitus in Patients With COngestve Heart Failure; UGDP=University Group Diabetes Program; UKPDS=United Kingdom Prospective Diabetes Study; VACSDM=Veterans Affairs Cooperative Study in Type 2 Diabetes Mellitus; VADT=Veterans Affairs Diabetes Trial. 


\section{Figures}

\section{Corrected Figure 10}

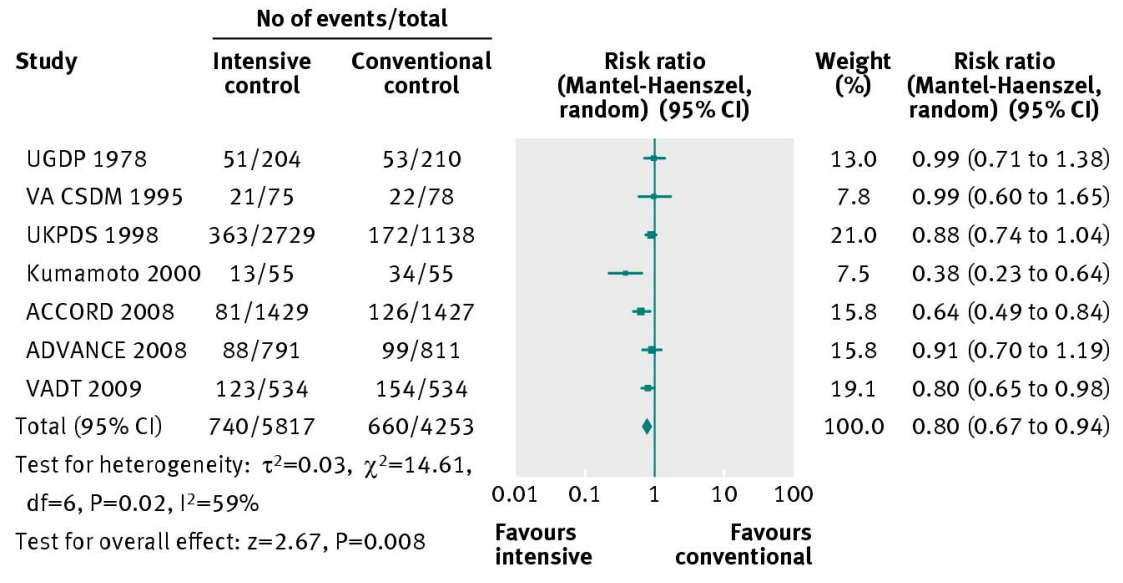

\section{Corrected Figure 11}

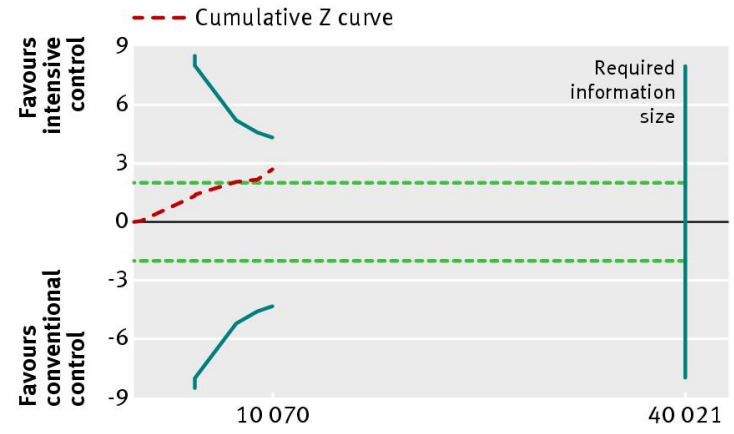

No of patients (linear scaled) 